\title{
UAV Fleet Mobility Model with Multiple Pheromones for Tracking Moving Observation Targets
}

\author{
Christophe Atten ${ }^{1}$, Loubna Channouf ${ }^{2}$, Grégoire Danoy ${ }^{2}$, and Pascal Bouvry ${ }^{2}$ \\ 1 SnT Interdisciplinary Centre, University of Luxembourg, Luxembourg \\ christophe.atten.001@student.uni.lu \\ 2 CSC Research Unit, University of Luxembourg, Luxembourg \\ loubna. channouf.001@student.uni.lu \\ \{gregoire.danoy, pascal.bouvry\}@.uni.lu
}

\begin{abstract}
The last years, UAVs have been developed to address a variety of applications ranging from searching and tracking to the surveillance of an area. However, using a single UAV limits the range of possible applications. Therefore, fleets of UAVs are nowadays considered to work together on a common goal which requires novel distributed mobility management models. This work proposes a novel nature-inspired mobility model for UAV fleets based on Ant Colony Optimisation approaches (ACO). It relies on two types of pheromones, a repulsive pheromone to cover the designated area in an efficient way, and an attractive pheromone to detect and to track the maximum number of targets. Furthermore, all decision takings are taken online by each UAV and are fully distributed. Experimental results demonstrate promising target tracking performances together with a small increase in the exhaustivity of the coverage.
\end{abstract}

Keywords: UAV, Mobility Model, Pheromone, Target Tracking, Ant Colony Optimisation

\section{Introduction}

Airborne surveillance of an area or tracking of some moving targets are typical military applications. However, these are also applicable in various civilian domains, from search and rescue to forest fire detection and water pollution detection. The usage of unmanned aerial vehicles (UAVs) for such applications has seen a dramatic increase in the past years. Current solutions typically rely on a single UAV, which restricts the observation capabilities and range of action due to a limited payload capacity and autonomy. In addition tracking can only be achieved on a single target.

Using several UAVs, referred to as a fleet, equipped with wireless communication capabilities permit to address these limitations. These have been recently considered for various applications, ranging from forest fires surveillance [1] to forest environment assessment [2] and emergency management in crisis zones [3]. 
Such communicating UAVs fleets constitute a specific type of mobile ad hoc network (MANET) referred to as Flying Ad Hoc Networks (FANETs). This work specifically considers a fleet of autonomous fixed-wings UAVs equipped with communication and sensing (e.g. radar) hardware. Operating fleets of autonomous UAVs raises novel issues such as mobility management and communication maintenance with the base station.

We here propose a novel nature-inspired mobility model, based on Ant Colony Optimisation (ACO), that extends the surveillance-oriented mobility model introduced by Schleich et al. in [4]. In the latter, the UAVs could perform an efficient scanning of an area by dropping repulsive pheromones on already scanned zones. The objective was to prevent other UAVs of the fleet to visit recently scanned areas. In order to fulfil the mission of target discovery and tracking, a second type of digital pheromones, i.e. attractive pheromones, is added in this contribution. These will help the UAVs to follow the targets by dropping such attractive pheromones where targets were discovered. As pheromones evaporate in time as in nature, UAVs can only use them during a limited amount of time to find back lost targets.

The remainder of this paper is organised as follows. In Section 2 the related work is presented, followed by a detailed and formalised problem description in Section 3. Then, in Section 5, the different quality metrics to assess the algorithm's performance are presented. The proposed novel mobility model with two types of pheromones is described in Section 4. Section 6 and 7 respectively present the setup used for the evaluation of the different scenarios and the analysis of the numerical results. Finally, Section 8 provides some conclusions and future perspectives.

\section{Related Work}

The UAV field has seen a tremendous growth in the last years which raises new issues, from legal aspects such as flight regulations, to R\&D perspectives such as autonomous swarms management [5].

This work focuses on mobility management of autonomous UAV swarms in the context of area surveillance, target detection and tracking. UAVs are equipped with wireless networking interfaces that allow them to communicate in an ad hoc fashion, forming so called Flying Ad hoc NETworks (FANETs).

In order to benefit from the full flexibility offered by autonomous UAV swarms, online and distributed path planning methods are desirable. However most existing works consider either centralised methods or distributed ones but using offline pre-computation and online decision taking $[6,7]$.

Few works proposed both distributed and online methods. One promising approach is ant colony, a nature-inspired method that relies on stigmergy. Ants deposit virtual pheromones in the environment, e.g. to indicate recently explored regions. As in nature, these pheromones will then evaporate in time.

This work proposes to extend one such pheromone-based mobility model [8], that uses digital repulsive pheromones for surveillance. This way the UAVs 
perform an efficient coverage by scanning in priority areas that contain no or a small amount of pheromones.

Some evolutions of pheromone-based models were proposed, such as [9] which performance was demonstrated against a random mobility model for surveillance, target acquisition and tracking.

Nevertheless, network connectivity was still presented as a problem. This was addressed in [4] which focused on using multiple UAVs for area coverage with a connectivity constraint. The latter work however focused only on a surveillance scenario and used only one type of pheromone, called repulsive pheromone. A main characteristic of this pheromone is to lead other UAVs in the fleet to other places in the area which are not controlled yet. This strategy leads to a higher coverage of the aforementioned area. In addition, this model served as basis for the pheromone model proposed in this paper, which will use a supplementary type of digital pheromone in order to fulfill the mission of target discovery and tracking.

A general multiple pheromone approach is described in [10], in which different rules can be created for the different pheromones. However, this was used in a different context, i.e. for classification. The algorithm designed in this work is based on a second type of pheromone, an attractive one for target discovery and tracking, along with the already existing repulsive pheromone used for surveillance.

\section{Problem Description}

This work aims at optimising the mobility of swarms of autonomous UAVs in a distributed and online fashion for both area surveillance and targets tracking while maintaining the UAV network connectivity. The latter goal is important for safety reasons as it is generally required by air regulations to be able to bring a flying entity back to the base station at any time. The considered UAVs are fixed-wing units which payload embeds wireless communication interfaces, allowing to send and receive message from/to the base station and other UAVs in an ad hoc fashion, and a scanner that permits to cover some geographical area.

The focus is put on the following three main objectives to appraise the quality of the proposed mobility models:

- The exhaustivity of the coverage: covering a maximum of the surveilled area.

- The global connectivity between the UAVs and base station reachability.

- The observation of targets in the surveyed area are composed of target tracking, untracked targets and target detection.

\section{Multiple Pheromone UAV Mobility Model}

In the following section the main contribution of this work is provided, i.e. a UAV mobility model based on two different types of pheromones: repulsive pheromones 


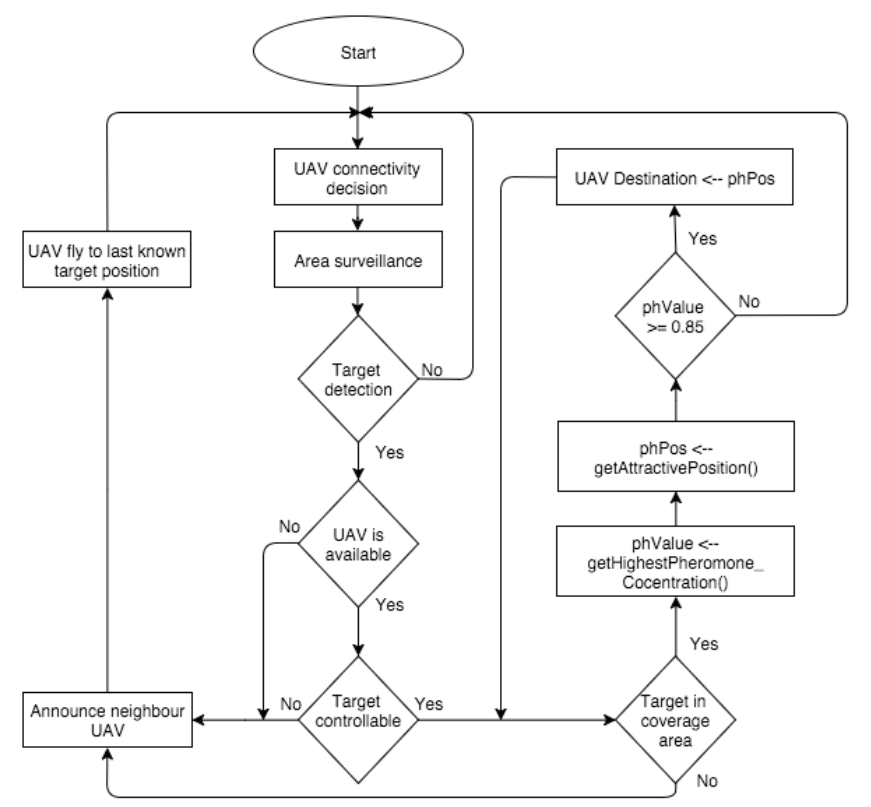

Fig. 1. Flowchart of the multiple pheromone mobility approach

to cover the area and attractive pheromones to follow targets. The repulsive pheromones allow UAVs to scan the area in an ant colony fashion avoiding to visit places that were covered recently, i.e. still containing repulsive pheromones. Attractive pheromones are deposited by UAVs that are tracking targets, so as to allow other UAVs to follow targets or to attract them in areas where the probability to find new targets is high.

This mobility model consists of the following sequential steps: connectivity approaches, area surveillance, target discovery, decision taking based on the UAV state and position, following best attractive positions and finally, target loss and recovery. The corresponding flowchart is presented in Figure 1. Each step is described in detail in the following subsections.

\subsection{Connectivity Approaches}

The proposed mobility model is based on multiple pheromones. It thus uses the idea of stigmergy, i.e. the ability to coordinate agents in an indirect fashion based on the exchange of information (pheromones) in the environment. In our scenario, the environment, i.e. the area to be scanned, cannot be used for exchanging pheromones. As a result, we rely on a virtual and discrete representation of the territory via a map.

Furthermore, two different UAV connectivity approaches are considered. The first variant, the UAV Inter-communication with usage of unavailable neighbour $U A V s$, referred to as maximum connectivity, focuses on staying connected as 

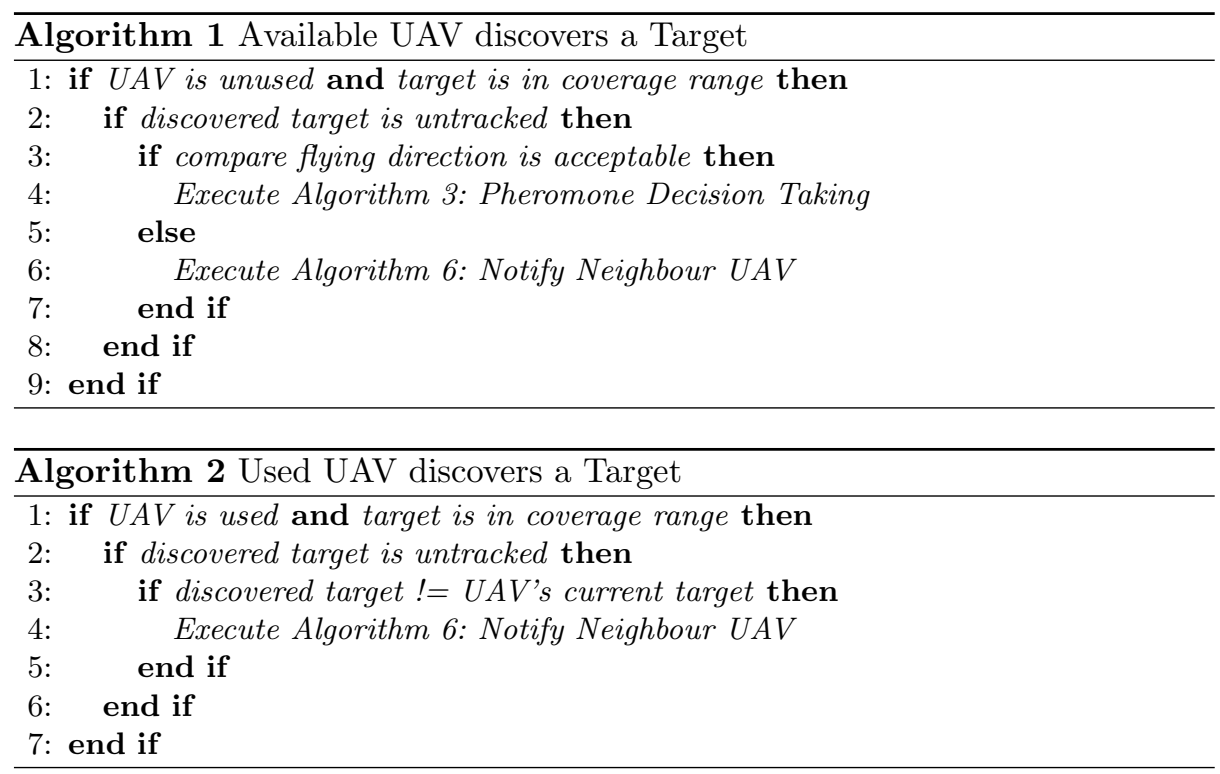

much as possible to cover the area, even if a neighbour UAV is currently following a target and not used to cover the area only. All the neighbour UAVS are used, which puts emphasis on the connectivity, but potentially to the expense of the area coverage. The second variant, the $U A V$ Inter-communication without usage of unavailable neighbour UAVs, referred to as low connectivity, ignores the UAVs which are following targets for building a connected UAV fleet group. The latter could lead to a worse connectivity of the overall fleet, but might improve the coverage of the area and optimise the following of the targets, as the UAVs would be more spread. Both models have a so-called critical point that occurs when a UAV is following a target. The latter will choose at every time-step a new destination based on the attractive pheromones to optimise the following of the targets.

\subsection{Area Surveillance}

By default, all UAVs scan the area in order to detect targets. The UAVs scanning approach is similar to the one previously proposed for area surveillance [4]. It relies on repulsive pheromones, evaporating in time, that indicate recently visited geographical areas. Each UAV then moves left, right, or forward, with a probability that is inversely proportional to the amount of pheromones in these places.

\subsection{Target Detection}

In order to ensure target discovery and tracking, the mobility model was extended with a second type of pheromone, referred to as attractive pheromone. 


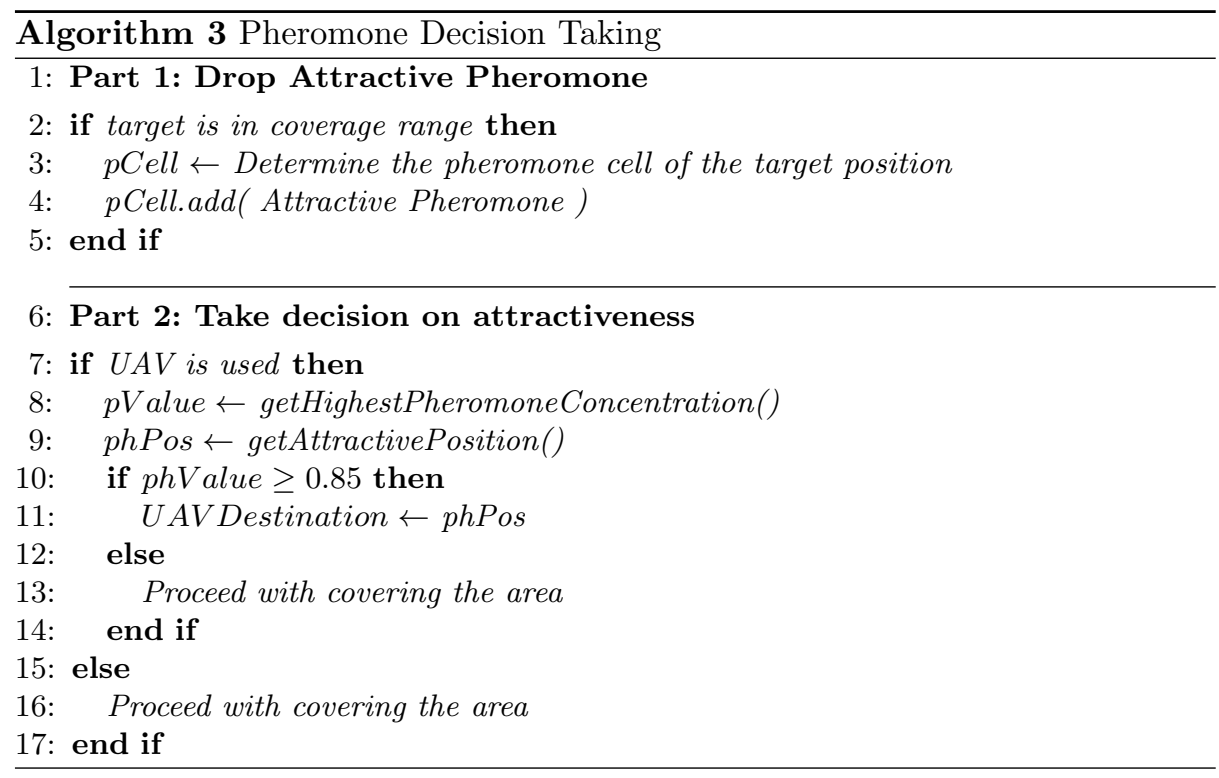

The discovery of a target is described in Algorithm 1 and Algorithm 2 for an available UAV or used UAV (i.e. already following a target) respectively. These are placed where and when a target is discovered and spread as long as it is followed by a UAV, as described in Algorithm 3.

\subsection{Following a Target Based on Attractiveness}

Target tracking is defined by how a UAV decides to follow or to cover the area for new targets. This is described in Algorithm 3. Part 1 of the algorithm describes the deposit of attractive pheromones. These are placed on cells that compose the discretised geographical area, a single cell being described as pCell. The second part of the algorithm describes the decision taking between either covering or tracking. When the concentration of attractive pheromones, phValue, in one or more neighbouring cells (front, left and right) is higher than 0.85 (85\%), the UAV chooses the position of the highest attractive pheromone concentration, phPos, as new destination. If the concentration is lower than 0.85 in the three neighbouring cells, the UAV will continue with covering the area.

\subsection{Computing Best Decision}

Fixed-wing UAVs cannot hover nor change instantly their direction to follow every discovered target. It might then happen that a UAV cannot follow one detected target. At each iteration a UAV has then to decide whether to keep following the target or to notify a neighbour UAV that is a good candidate for tracking the considered target. For this, three algorithms are presented to illustrate this decision taking. 

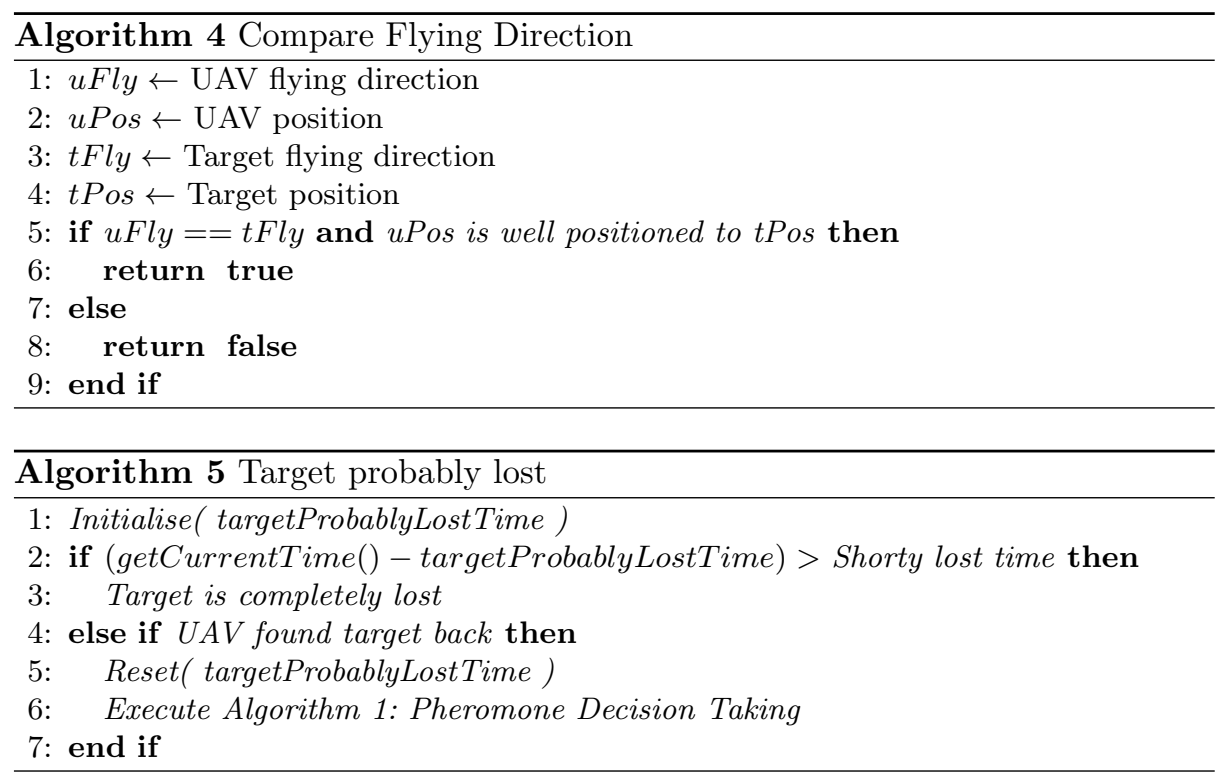

Algorithm 4 shortly describes the verification if a UAV is well-positioned to follow a discovered target. In more details, when a UAV discovers a target, it compares its position and flying direction to the target ones. For instance, when the UAV is behind the target and both the UAV and target are flying to the North, the UAV is well positioned to follow the target.

This algorithm will search for the closest neighbour UAV which is available, which means, when a UAV is not used to track a target. This UAV is then notified to fly to the last known position of the target.

\subsection{Target Loss and Recovery}

The last part of this new mobility model deals with the case when a UAV lost a target and wants to find it back. Algorithm 5 shortly describes the usage of a new parameter, i.e. the time a target is shortly lost. This parameter is added to give a UAV a chance to find the target back after loosing it. If the UAV finds the target back, the tracking is not interrupted, otherwise its is interrupted and Algorithm 6 is executed. When the UAV has definitively lost the target, the amount of time a UAV probably lost a target until the time the target is definitively lost, will be substracted from the average tracking time.

\section{Quality Metrics}

This section introduces the different metrics used to assess the performance of the proposed mobility models, both in terms of surveillance and target observation. The first three metrics are related to the observation and networking objectives, 


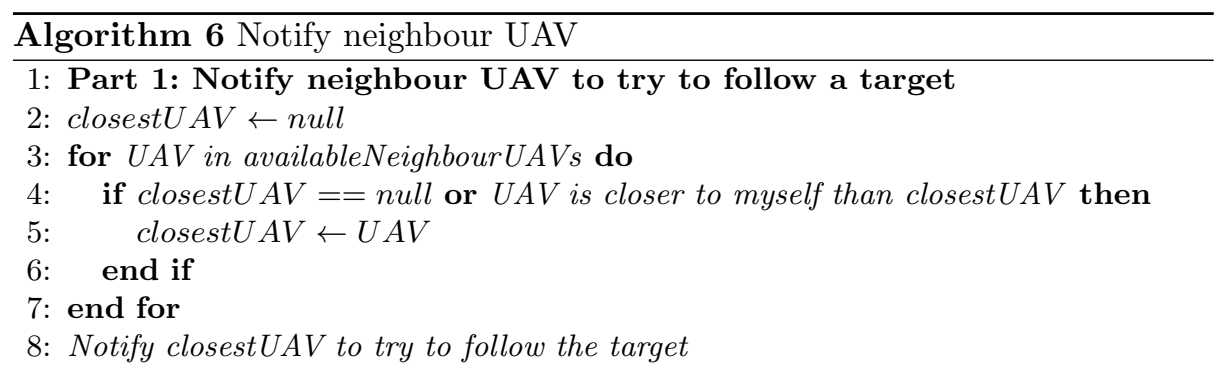

9: Part 2: Receiving order to control an unreachable target

10: if $U A V$ got message from neighbour to control an unreachable target then

11: Unused UAV will fly to the last known position of this target

12: if $U A V$ discovers a target then

13: $\quad$ Execute Algorithm 1: Available UAV discovers a Target

14: else

15: $\quad$ Unused UAV will continue normal patrolling

16: end if

17: end if

namely the exhaustivity of the coverage, the global connectivity between the UAVs and the reachability of the base station. The other three metrics permit to evaluate the targets observation and tracking abilities, i.e. the average and best amount of time a target is tracked, the amount of time a target is untracked and the number of target detections.

\subsection{Exhaustivity of the Coverage}

The quality of the geographical area coverage is expressed by the exhaustivity. It describes the amount of area that has never been scanned during a simulation run. Literally, it is the percentage of never scanned cells in the discretised area. More information about the exhaustivity of the coverage can be found in [4]. As a complement, the percentage of scanned cells during the simulation is also measured.

\subsection{Connectivity}

Two types of connectivities are considered, i.e. between UAVs and between UAVs and the base station. The number of connected components grants information about how much disconnected is a graph, i.e. how many groups of UAVs exist. The optimal value for this metric is thus 1 . Moreover, the number of connections to the base station is represented in percentage. This value provides information on how many UAVs can communicate over one or multiple hops to the base station. 


\subsection{Average and Longest Target Tracking Time}

The target tracking time is the amount of time a UAV manages to follow a target it has detected. This metric is calculated for all targets and represented as the average in milliseconds. The longest tracking time metric complements the previous one. It measures the longest time a UAV was able to follow a target during a run. This metric shows the average of all the longest tracking times in milliseconds.

\subsection{Target untracked Time}

While some UAVs successfully fulfil their mission of tracking targets, others may not be able to do so. Target unattainability is an inevitable consequence of a low amount of UAVs and respectively high amount of targets. The untracked time starts when a target status is undetected and ends when a UAV discovered it. This metric represents the average time during which targets are untracked by a UAV.

\subsection{Number of Target Detections}

The number of target detections is an important metric to consider in combination with the target untracked time and the tracking time. This metrics is represented as the total amount of detections in a run.

\section{Experimental Setup}

In this section, the simulation environment is presented along with the most important experimental parameters. Afterwards, the two variants of the mobility model for the UAVs are presented as well as the single pheromone mobility

model these are compared to. Finally, the two mobility models for the targets are introduced.

\subsection{Simulation Environment}

The simulation environment is fully based on the dynamic graph library used in [4]. In this subsection we provide details concerning the implementation of the area, the autopilot of the UAVs and the main simulation parameters which values are summarised in Table 1.

The Area The simulation area is a rectangle with a long side $L_{l o n g}$, short side $L_{\text {short }}$ and discretised in square cells of side $L_{\text {cell }}$ as in [4]. Additional parameters are required when the Manhattan mobility model is used for the targets (described in section 6.3). $M B_{\text {width }}$ and $M B_{\text {height }}$ describe the size of a Manhattan box, which is a square of dimension $M B_{\text {width }} * M B_{\text {height }}$. Hence, the quantity of Manhattan boxes in the simulation area $L_{\text {long }}$ and $L_{\text {short }}$ is:

$$
M B_{\text {quantity }}=\frac{L_{\text {long }} * L_{\text {short }}}{M B_{\text {width }} * M B_{\text {height }}}
$$


Table 1. Main simulation parameters

\begin{tabular}{|c|c|c|c|}
\hline Simulation Area & Parameter Value & Target Autopilot & Parameter Value \\
\hline$L_{\text {long }}$ & 2000 & Speed & {$\left[\begin{array}{lll}0 & . . & 5\end{array}\right]$} \\
\hline$L_{\text {short }}$ & 1000 & Acceleration & {$\left[\begin{array}{lll}-1 & . . & 1\end{array}\right]$} \\
\hline$L_{\text {cell }}$ & 20 & Heading change & {$\left[\begin{array}{lll}0 & . . & 0.1\end{array}\right]$} \\
\hline Base station position & $(1000,0)$ & Decision Frequency & {$[1,30]$} \\
\hline UAV Autopilot & Parameter Value & Shortly lost time & $500 \mathrm{~ms}$ \\
\hline \multirow{2}{*}{$\begin{array}{l}\text { Speed } \\
\text { Acceleration }\end{array}$} & {$\left[\begin{array}{lll}0 & . . & 5\end{array}\right]$} & Manhattan Grid & Parameter Value \\
\hline & {$\left[\begin{array}{lll}-1 & . . & 1\end{array}\right]$} & $M B_{\text {width }}$ & 200 \\
\hline \multirow{2}{*}{$\begin{array}{l}\text { Heading change } \\
\text { Decision Frequency }\end{array}$} & {$\left[\begin{array}{lll}0 & . . & 0.1\end{array}\right]$} & $M B_{\text {height }}$ & 100 \\
\hline & {$[1,30]$} & \begin{tabular}{|l|} 
Experiments \\
\end{tabular} & Parameter Value \\
\hline Future & 30 & Number of UAVs & {$\left[\begin{array}{lll}10 & . . & 50\end{array}\right]$} \\
\hline Wireless Range & 400 & Number of runs per experiment & 30 \\
\hline Coverage Range & 2 & Number of Targets & 10 \\
\hline Attractive Pheromone Thre & $0.85(85 \%)$ & & \\
\hline
\end{tabular}

UAV Autopilot The implemented autopilot for the fixed-wing UAVs is similar to [4] in case of area surveillance, with a destination change every 30 time-steps. However, in order to ensure a higher accuracy when tracking targets, the current destination is updated every time-step. This is motivated by the criticality of this phase for the UAVs that need to react fast to target destination changes.

Simulation Parameters As all the mobility models are stochastic, each set of experiments was repeated 30 times in order to obtain a good statistical confidence. For all quality metrics the average was computed. The results obtained on the 8 scenarios have been statistically compared according to the Wilcoxon unpaired signed rank test for the 8 metrics [11]. As it can be seen in Table 1, experiments were conducted with very different density conditions, ranging from 10 UAVs up to 50 with a wireless transmission range fixed at 400 .

Limitations As the UAV model is based on [4], the limitations are also similar. Additionally, it is assumed that every UAV knows if a neighbour UAV is busy or not. This information could get exchanged in a real-world execution through so-called beaconing.

\subsection{Compared UAV Mobility Models}

The multiple pheromone mobility model is compared to the repulsive pheromone mobility model from [4]. In this single pheromone mobility model, each UAV locally decides where to move based on different criterias, such as future position of one-hop neighbors, as well as the concentration of the repulsive pheromone around, to attract UAVs to places that are characterised by the lowest pheromone concentration. The target detection has no direct impact on the movement of the old mobility model proposed in [4] as long as there is no attractive pheromone 
Table 2. Possible scenarios with abbreviations

\begin{tabular}{|c|c|c|c|c|c|c|}
\hline \multicolumn{2}{|c|}{ UAV Connectivity } & \multicolumn{2}{|c|}{\begin{tabular}{|c||} 
UAV Model \\
Multiple Pheromones|Single Pheromone
\end{tabular}} & \multicolumn{2}{|c|}{\begin{tabular}{c|} 
Target Model \\
Random $\mid$ Manhattan
\end{tabular}} & $\mid \begin{array}{c}* \mathrm{MC}=\text { Maximum Connectivity } \\
\mathrm{LC}=\text { Low Connectivity } \\
\text { Abbreviation }\end{array}$ \\
\hline $\mathrm{X}$ & & $\mathrm{x}$ & & $\mathrm{X}$ & & MCMR \\
\hline $\mathrm{X}$ & & $\mathrm{X}$ & & & $\mathrm{X}$ & MCMM \\
\hline $\mathrm{X}$ & & & $\mathrm{x}$ & $\mathrm{X}$ & & MCSR \\
\hline $\mathrm{x}$ & & & $\mathrm{X}$ & & $\mathrm{X}$ & MCSM \\
\hline & $\mathrm{X}$ & $\mathrm{x}$ & & $\mathrm{X}$ & & LCMR \\
\hline & $\mathrm{X}$ & $\mathrm{X}$ & & & $\mathrm{X}$ & LCMM \\
\hline & $\mathrm{X}$ & & $\mathrm{X}$ & $\mathrm{X}$ & & LCSR \\
\hline & $\mathrm{x}$ & & $\mathrm{X}$ & & $\mathrm{X}$ & LCSM \\
\hline
\end{tabular}

around. Indeed, the attractive pheromone has a higher priority than the repulsive one.

\subsection{Target Mobility Models}

Two different target mobility models are used, a random mobility model and a Manhattan mobility model. The random mobility model decides uniformly at random between three possible directions with some fixed probabilities: front $(60 \%)$, right $(20 \%)$ and left $(20 \%)$. So each target randomly chooses a new direction (Left, Front, Right). This decision is taken every 30 time-steps when the target is untracked. If the target is tracked, it will switch to the critical mode, where the decision is made every time-step. The second mobility model, Manhattan mobility model, is based on a grid of "streets", that represents a simplified city road topology. Targets mobility is constrained on these streets. The dimension of the grid is expressed in Table 1 . Targets have again three direction choices, i.e. left, front or right, with $25 \%, 25 \%$ and $50 \%$ probabilities respectively. Decisions can only be made on crossroads. Furthermore, the critical mode is not available by the Manhattan mobility model through the fact that the targets can only decide on corner points.

\section{$7 \quad$ Numerical Results}

This section presents the evaluation and comparison of the different mobility models using the metrics presented in Section 5 and Table 2.

\subsection{Coverage and Connectivity}

Number of Scanned Cells. For the number of scanned cells, presented in Figure 2, it can be observed that the new multiple pheromones UAV models, LCMM, LCMR, MCMM and MCMR are performing slightly better than the other ones, especially in the densest scenarios. This outlines that the usage of the two pheromone types permits the UAVs to reach unvisited places faster. 


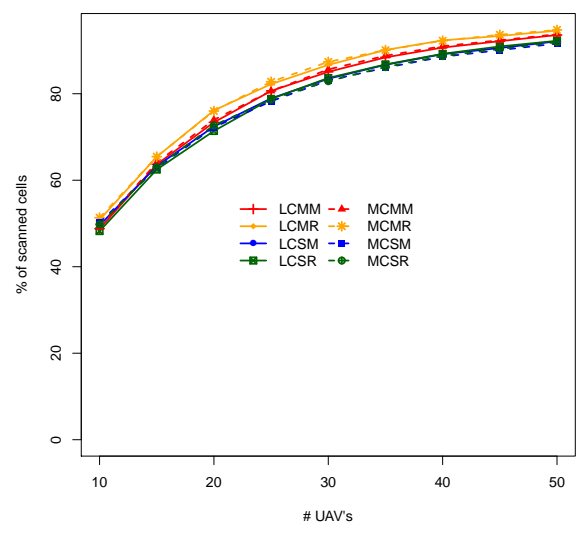

Fig. 2. Percentage of scanned cells

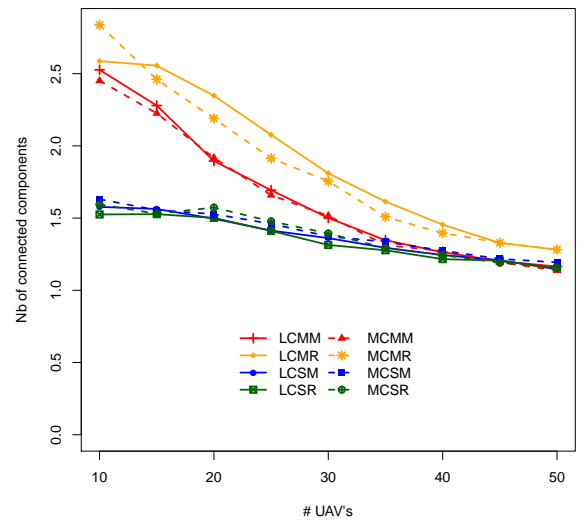

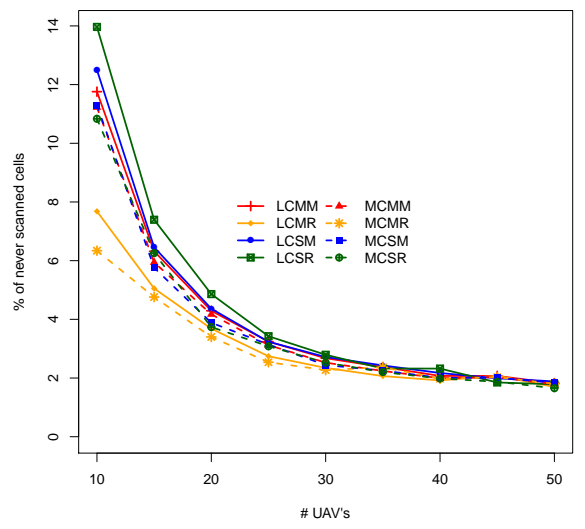

Fig. 3. Percentage of never scanned cells

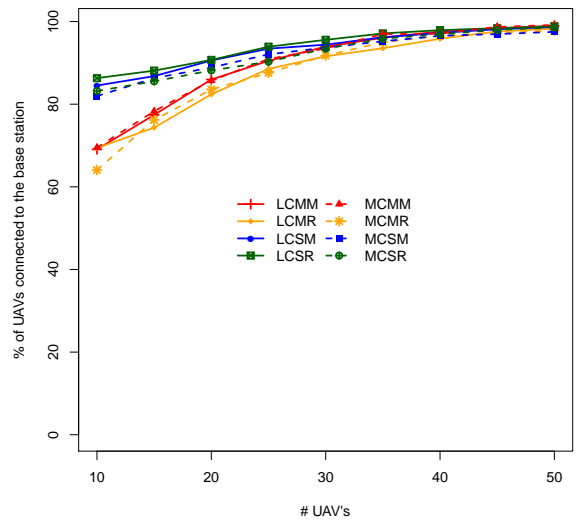

Fig. 4. Number of connected components Fig. 5. UAVs connected to the base station

Furthermore, we can claim with statistical confidence that the usage of two types of pheromones, MCMR and LCMR, provides the best results.

Number of Never Scanned Cells. The number of never scanned cells in Figure 3 confirms the previous results. More scanned cells logically implies less never scanned cells. For the lowest density, i.e. $10 \mathrm{UAVs}$, LCMR and MCMR perform best with statistical confidence on the random mobility targets scenario with values ranging between 6 and $8 \%$, which is two times better than the previous approaches. Similarly, the multiple pheromone models, LCMR and MCMR, perform better on the random mobility targets than the Manhattan approach with statistical confidence. This advantage decreases as the UAVs density increases, which logically means that the area is better covered. 


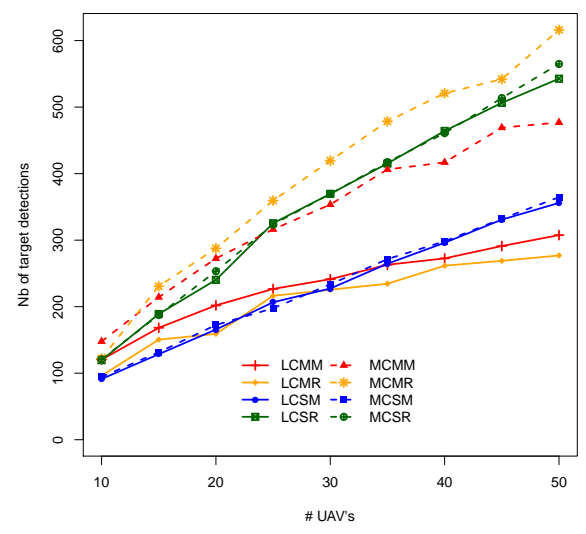

Fig. 6. Number of target detections

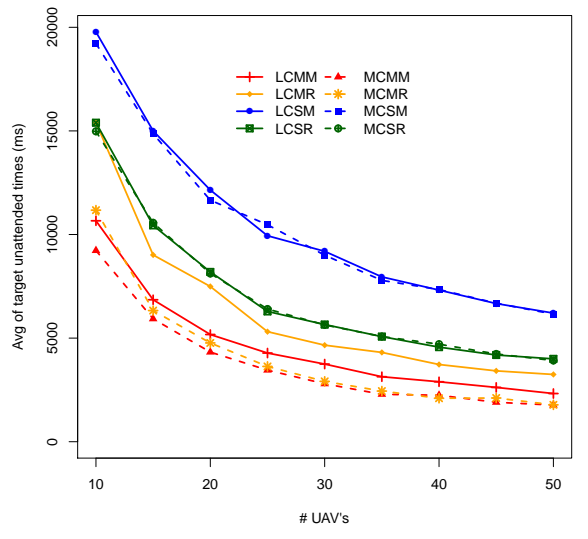

Fig. 7. Average untracked time

Number of Connected Components. This metrics represents the number of connected components (groups) during the simulation see Figure 4. The optimal value is 1 , which means that the base station can reach any UAV using multi-hop communication. At the connectivity level, we can say with statistical confidence, that the multiple pheromone approaches perform less good than the single pheromone approaches. This is not very surprising since the target following and the higher priority of the attractive pheromones imply that UAVs are more distributed in the simulation area.

Number of Components Connected to the Base Station. Similarly to the number of connected components, we can say with statistical confidence that the multiple pheromone approaches are performing less good at a low density of UAVs, which is not very surprising since the target following has a higher priority, as presented in Figure 5. However, for all number of UAVs we can say that there is no statistical difference between all scenarios.

\section{2 $\quad$ Tracking}

Number of Detections. For the number of detections in Figure 6 we can say that a lower number of detections might reflect to higher observation times while following a target. Nevertheless, it could also lead to high times for a few untracked targets, while some UAVs are actually following their target and the rest of the fleet is not able to track down the remaining targets.

Average Untracked Time of a Target. It clearly appears with statistical confidence that the multiple pheromone models always outperform the random 


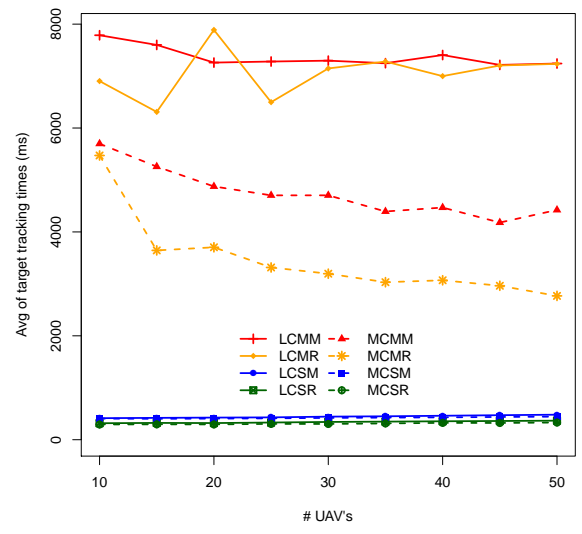

Fig. 8. Average tracking times

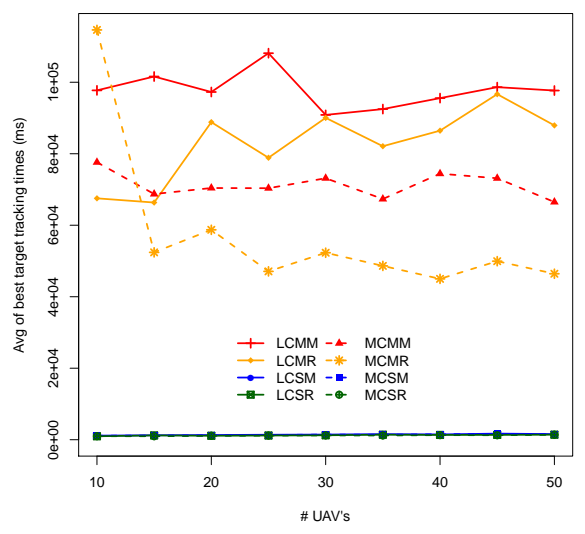

Fig. 9. Average of longest tracking times

ones on all scenarios, i.e. LCMM, MCMM, LCMR and MCMR, see Figure 7. The maximum connectivity approaches, MCMM and MCMM provide better results with statistical confidence to other scenarios, which means that UAVs flying in a more connected way are able to better track targets back. This difference remains the same no matter the UAV density.

Average Tracking Time of a Target. In Figure 8 we can observe with statistical confidence that the multiple pheromone models with the low connectivity approach, i.e. LCMM and LCMR provide the best results on the two scenarios, with an average of $7200 \mathrm{~ms}$. This result is around $4100 \mathrm{~ms}$ better than by using the maximum connectivity variant, which is logical, since in that case UAVs that are tracking targets might loose them to remain connected with the swarm. A notifiable difference exists between the two target mobility models. The Manhattan model is more continuous and stable. This is explained by the fact that targets move on streets and not randomly in the whole area. With statistical confidence we can say that UAVs perform better on the Manhattan scenario model in comparison to the random one. This can be explained by the random model properties, that permits targets to move shortly out of the area covered by the UAVs. Targets will thus be lost since the UAVs cannot put attractive pheromones outside of the area, nor follow an attractive pheromone path.

Average of the Best Tracking Times of a Target. In Figure 9, we can see the average of the best following times for a UAV. With statistical confidence, LCMM is in average performing better due to the fact of the higher distribution in the area, which leads to a possible higher number of detections and possible better positioning in the area to follow a target and the more continuous moving of the targets without leaving the area. 


\subsection{Summary}

These experiments have provided information about the performance of the different UAV mobility models and associated connectivity approaches on two target models, i.e random and Manhattan. For both coverage and tracking, the multiple pheromone models combined with the low connectivity strategy, LCMM and LCMR, provide the best results in most cases compared to the MCMM, MCMR or the random mobility model without tracking MCSM, MCSR, LCSM and LCSR. Mainly, LCMM and LCMR reach very good results for target tracking and even better results for coverage compared to their competitors. Nevertheless, these results induce a small degradation in terms of connectivity between the UAVs and the base station. This drawback is not a surprise, because the UAVs first priority is the tracking of targets. Therefore, this slightly worse connectivity is not too penalising since much better results are obtained in targets tracking.

\section{Conclusion and Perspectives}

This paper proposed a novel decentralised and online mobility model with multiple pheromones for surveillance and target tracking missions. The latter consists in the combination of a repulsive pheromone-based mobility model [4] with an attractive pheromone one. On the one hand, the repulsive pheromones are used to survey the area by repelling UAVs to less scanned cells, and on the other hand, the attractive pheromones are used to follow and track discovered target. Its performance is evaluated using a set of state-of-the-art and novel quality metrics. With this combination, the proposed mobility model was able to outperform a single pheromone mobility model extended with the proposed connectivity approaches in terms of coverage of the area (i.e repulsive pheromone) and showed good target tracking abilities.

Future work will focus on extending the multiple pheromone mobility model with target path predictions in case of target loss as well as trying to adapt the threshold for the attractive pheromone. Moreover, we can implement a multilevel UAV swarm with different types of UAVs as in the article [12], to increase the connectivity stability and achieve possible better tracking times.

\section{References}

1. Casbeer, D.W., Kingston, D.B., Beard, R.W., McLain, T.W.: Cooperative forest fire surveillance using a team of small unmanned air vehicles. Int. J. Systems Science 37 (2006) 351-360

2. Brust, M.R., Strimbu, B.M.: A networked swarm model for UAV deployment in the assessment of forest environments. In: Tenth IEEE International Conference on Intelligent Sensors, Sensor Networks and Information Processing, ISSNIP 2015, Singapore, April 7-9, 2015, IEEE (2015) 1-6

3. Bupe, P., Haddad, R., Rios-Gutierrez, F.: Relief and emergency communication network based on an autonomous decentralized uav clustering network. In: SoutheastCon 2015. (2015) 1-8 
4. Schleich, J., Panchapakesan, A., Danoy, G., Bouvry, P.: UAV fleet area coverage with network connectivity constraint. In: Proceedings of the 11th ACM International Symposium on Mobility Management and Wireless Access. MobiWac '13, New York, NY, USA, ACM (2013) 131-138

5. Valavanis, K.P., Vachtsevanos, G.J.: Handbook of Unmanned Aerial Vehicles. Springer (2015)

6. Althoff, D., Althoff, M., Scherer, S.: Online safety verification of trajectories for unmanned flight with offline computed robust invariant sets. In: IEEE/RSJ International Conference on Intelligent Robots and Systems. (2015)

7. de la Cruz, J.M., Besada-Portas, E., Torre-Cubillo, L., Andres-Toro, B., LopezOrozco, J.A.: Evolutionary path planner for uavs in realistic environments. In: Proceedings of the 10th Annual Conference on Genetic and Evolutionary Computation. GECCO '08, New York, NY, USA, ACM (2008) 1477-1484

8. Simon, D.: Evolutionary Optimization Algorithms. Wiley (2013)

9. Kuiper, E., Nadjm-Tehrani, S.: Mobility models for UAV group reconnaissance applications. In: Wireless and Mobile Communications, 2006. ICWMC '06. International Conference on. (2006) 33-33

10. Salama, K.M., Abdelbar, A.M., Otero, F.E.B., Freitas, A.A.: Utilizing multiple pheromones in an ant-based algorithm for continuous-attribute classification rule discovery. Appl. Soft Comput. 13 (2013) 667-675

11. Wilcoxon, F.: Individual Comparisons by Ranking Methods. Biometrics Bulletin 1 (1945) 80-83

12. Danoy, G., Brust, M.R., Bouvry, P.: Connectivity stability in autonomous multilevel uav swarms for wide area monitoring. In: Proceedings of the 5th ACM Symposium on Development and Analysis of Intelligent Vehicular Networks and Applications. DIVANet '15, New York, NY, USA, ACM (2015) 1-8 